\title{
1 Literarische Wissenschaftsgeschichtsschreibung zwischen Geschichtswissenschaft und Wissenschaftsgeschichte
}

\subsection{Kehlmanns wissenschaftshistorischer Roman Die Vermessung der Welt als Sonderfall des historischen Romans}

\begin{abstract}
Im September 1828 verließ der größte Mathematiker des Landes zum erstenmal seit Jahren seine Heimatstadt, um am Deutschen Naturforscherkongreß in Berlin teilzunehmen. Selbstverständlich wollte er nicht dorthin. Monatelang hatte er sich geweigert, aber Alexander von Humboldt war hartnäckig geblieben, bis er in einem schwachen Moment und in der Hoffnung, der Tag käme nie, zugesagt hatte. ${ }^{1}$
\end{abstract}

Diese Sätze, mit denen Daniel Kehlmanns im Jahr 2005 erschienener Roman Die Vermessung der Welt beginnt, weisen eine für den historischen Roman ${ }^{2}$ charakteristische Doppelung von Erzählstruktur und Erzählfunktion auf: Während mit den konkreten Zeit-, Orts- und Ereignisangaben des ersten Satzes - verknüpft mit der „panoramatischen Zeitstruktur des Erzählerstandpunktes“3 - Parameter gesetzt sind, die den historischen Rahmen skizzieren und damit die „Funktion historisch individualisierender Orientierung und Determination “4 erfüllen, setzt sich im zweiten das „szenische, mit dem Personenstandpunkt gegebene ,Analogon der erlebten Zeit““5 durch, der die „Funktion fiktionalen Entwerfens“6 ${ }^{\text {" }}$ mar-

1 Daniel Kehlmann: Die Vermessung der Welt [2005], Reinbek b. Hamburg 22005, im Folgenden zitiert unter der Sigle V.

2 Vgl. dazu Hans Vilmar Geppert: Der ,andere' historische Roman. Theorie und Strukturen einer diskontinuierlichen Gattung, Tübingen 1976, sowie ders.: Geschichte umerzählt von Walter Scott bis zur Gegenwart, Tübingen 2009. Zur Deutung des Romananfangs vor dem Hintergrund der historischen Quellen vgl. Ina Ulrike Paul: Geschichte und Literatur Organon der Selbsterkenntnis. Über Daniel Kehlmanns Die Vermessung der Welt [2005], in: Der historische Roman zwischen Kunst, Ideologie und Wissenschaft, hrsg. v. Ina U. Paul u. Richard Faber, Würzburg 2013, S. 174 f.). - Kehlmann selbst dementiert die Zugehörigkeit seines Romans zur Gattung des historischen Romans, verbindet diese jedoch generell mit dem „Genre der Trivialliteratur“ (Daniel Kehlmann/Sebastian Kleinschmidt: Requiem für einen Hund. Ein Gespräch, Reinbek b. Hamburg ${ }^{2} 2018$, S. 71).

3 Geppert: Der ,andere‘ historische Roman, S. 21.

4 Ebd., S. 23.

5 Ebd., S. 21.

6 Ebd., S. 23.

D Open Access. (C) 2021 Bernadette Malinowski, publiziert von De Gruyter. (cc) BY-NC-ND Dieses Werk ist lizenziert unter der Creative Commons Attribution-NonCommercial-NoDerivatives 4.0 International Lizenz. https://doi.org/10.1515/9783110642384-004 
kiert. Diese Akzentuierung des „Hiatus von Fiktion und Historie“7 - eine Formel, mit der Geppert das „Verhältnis der Abhebung des Historischen vom Fiktiven“ im ,anderen“ historischen Roman beschreibt - setzt sich im dritten Satz dergestalt fort, dass die fiktional-szenische Erzählsituation zwar beibehalten, mit dem Namen Alexander von Humboldt jedoch eine weitere historische Größe eingeführt wird, die rückwirkend die historisch-dokumentarische Funktion des ersten Satzes bestätigt und konkretisiert. Die Protagonisten werden damit von Anbeginn in ,characters in fiction“ und ,characters in history ${ }^{6}$ gespalten, gleichsam in der Differenz von Geschichte und Fiktion verortet und in dieser bzw. als diese Differenz unterscheidbar. ${ }^{9}$ Mit den Angaben „größter Mathematiker“, „Naturforscherkongreß“ und „Alexander von Humboldt“ wird darüber hinaus der historische Gegenstand der narrativen Darstellung als ein wissenschaftshistorischer präzisiert, mit der personalen Fokussierung auf Gauß und Humboldt das wissenschaftshistorische Geschehen biographisch gebunden. Die Eingangssätze akzentuieren folglich nicht nur die „produktive Differenz von historischem und fiktionalem Diskurs“, sondern spalten zugleich den historischen Diskurs in einen biographisch-individualgeschichtlichen, zeitgeschichtlichen und wissenschaftsgeschichtlichen Diskurs auf, ohne freilich deren immanenten Zusammenhang, gewährleistet durch die Unhintergehbarkeit des Narrativen, aufzulösen. Während es nun nachgerade zur gattungsspezifischen Thematik des historischen Romans seit seinen Anfängen im frühen 19. Jahrhundert gehört, das menschliche Individuum ,in seiner Position gegenüber der kontingenten Geschichte“ zu definieren und das „problematisch gewordene Verhältnis individueller und kollektiver Zeitdimensionen"10 in der Verschränkung von biographischer und geschichtlicher Dimension narrativ zu verarbeiten, ${ }^{11}$ ist die Allianz von politisch-gesellschaftlicher Zeitgeschichte und Wissenschaftsgeschichte und entsprechend von Historiographie und Wissenschaftshistoriogra-

7 Ebd., S. 34.

$8 \mathrm{Vgl}$. Edward M. Forster: Aspects of the novel [1927], in: ders.: Aspects of the novel and related writings, London 1974, S. 1-119, hier S. 30-39.

9 Diese einmal gesetzte Differenzierung ist auch durch das komplexe Spiel mit dieser Differenz (ihre zeitweilige Überformung einerseits, ihre beinahe Auflösung andererseits), das den weiteren Verlauf des Romans prägt, nicht mehr rückgängig zu machen.

10 Fabian Lampart: Zeit und Geschichte. Die mehrfachen Anfänge des historischen Romans bei Scott, Arnim, Vigny und Manzoni, Würzburg 2002, S. 24 u. 28.

11 „Strukturen individueller Entwicklung sind von zentraler Bedeutung für die Historisierung des Romans“ (ebd., S. 96). In seiner Diskussion der Rolle, die der Bildungsroman für die Herausbildung des historischen Romans innehat, definiert Lampart den historischen Roman treffend als „,,Roman der Bildung zur Wahrnehmung des Historischen““ (ebd., S. 103). 
phie eine keineswegs selbstverständliche. Der Grund dafür ist vor allem darin zu sehen, dass die Wissenschaftsgeschichte bis zur Entstehung der „Science in Context"“-Bewegung in den 70er Jahren des letzten Jahrhunderts entscheidend durch die Überlegenheit der Naturwissenschaften geprägt war, also wesentlich als eine „history of science“ aufgefasst wurde, derzufolge die Entwicklung wissenschaftlicher Theorien, Forschungspraktiken und Institutionen unabhängig von allgemeinhistorischen Kontexten stattfindet. Vor dem Hintergrund der bereits im ersten Teil dargelegten , antipositivistischen' Tendenzen in der Wissenschaftsgeschichte und mit Blick auf den für Kehlmanns Roman charakteristischen doppelten Hiatus von Historie und Fiktion sowie Wissenschaftshistorie und Fiktion soll die Frage nach dem Verhältnis von Geschichte bzw. Geschichtswissenschaft und Wissenschaftsgeschichte im Folgenden kurz erläutert werden.

\subsection{Geschichtswissenschaft - Wissenschaftsgeschichte}

Für die Tatsache, dass insbesondere in Deutschland Geschichtswissenschaft und Wissenschaftsgeschichte bis vor kurzem eine Koexistenz geführt haben, werden in der Regel zwei Hauptursachen verantwortlich gemacht: ${ }^{12}$ Zum einen fiel die Aufgabe der (Natur-)Wissenschaftsgeschichtsschreibung traditionell in das Ressort der Naturwissenschaftler selbst, zum anderen war die Geschichtswissenschaft lange Zeit auf das ,historistische Paradigma einer Politikgeschichte im nationalhistorischen Rahmen" ${ }^{13}$ verengt. Erst im Gefolge der 68er Debatten, die seitens der Geschichtswissenschaft eine „Pluralisierung von Themen und Methoden“ zur Folge hatten, seitens der Wissenschaftsgeschichte - insbesondere durch Thomas S. Kuhns Studie „The Structure of Scientific Revolutions“14 - eine Historisierung wissenschaftlicher Prozesse und damit die „Suche nach auch außerhalb der Naturwissenschaften liegenden Begründungszusammenhängen des wissenschaftlichen Wandels“, ${ }^{15}$ kommt es zu einer Annäherung beider Disziplinen. Damit ist ein dritter maßgeblicher Grund für die bis dahin „gegenseitige Ignoranz“ von Geschichtswissenschaft und Wissenschaftsge-

12 Vgl. dazu und im Folgenden Helmuth Trischler: Geschichtswissenschaft - Wissenschaftsgeschichte: Koexistenz oder Konvergenz? in: Beiträge zur Wissenschaftsgeschichte 22 (1999), S. 239-256.

13 Ebd., S. 241.

14 Thomas S. Kuhn: The Structure of Scientific Revolution, Chicago 1962; dt. Die Struktur wissenschaftlicher Revolutionen, übers. v. Kurt Simon, Frankfurt/M. 1967.

15 Trischler: Geschichtswissenschaft - Wissenschaftsgeschichte, S. 242. 
schichte angeführt: Bis zu der von Kuhn eingeläuteten „antipositivistischen Wende" ${ }^{\text {116 }}$ wurde Wissenschaftsgeschichte als Erfolgs- und Fortschrittsgeschichte betrieben, Wissenschaft als "prozessuale, positivistisch verfahrende Akkumulation von Ideen und Entdeckungen, die in Tempo und Richtung durch wissenschaftsimmanente Kriterien vorbestimmt war" ${ }^{17}$ bewertet. Nach Mittelstraß liegt dieser Art von Wissenschaftsgeschichtsschreibung ein Geschichtskonzept zugrunde, das durch die „,Eigenschaft des Fortschritts““ und - konsequenterund paradoxerweise - durch die „,Eigenschaft der Geschichtslosigkeit““ gekennzeichnet ist. ${ }^{18}$ Mag diese ,ahistorische Wissenschaftsgeschichtsschreibung seit Kuhn eine erhebliche Problematisierung erfahren haben, so rücken mit ihr doch Aspekte eines wissenschaftlichen Selbstverständnisses in den Blick, die an Aktualität und Relevanz weder für die wissenschaftliche Praxis noch für das öffentliche ,Image‘ der Wissenschaft eingebüßt haben. Ein Aspekt betrifft den ontologischen Status naturwissenschaftlicher Erkenntnisse: Wenn „wahre Sätze“ zum „geschichtslosen Bestand einer Wissenschaft“19 zu rechnen sind, die der „historischen Darstellung nicht bedürfen“, ihre „falschen Sätze, Unklarheiten, Mißverständnisse etc. dagegen zur Historie“ gehören, folgerichtig „das Historische [...] für die Wissenschaft selbst das Uninteressante“ ist, dann wird die Wissenschaft selbst in eine ahistorische Wissenschaft und eine historische Nicht-Wissenschaft gespaltet und die Auffassung von Wissenschaft als teleologischer - gleichsam außerhalb der Geschichte verlaufender - Fortschrittsprozess erneut legitimiert. Eine ähnliche Problematik spiegelt sich in der Debatte ,Externalismus versus Internalismus $:^{60}$ Der externalistische Ansatz erklärt wis-

16 Kurt Bayerts: Wissenschaft als historischer Prozeß. Die antipositivistische Wende in der Wissenschaftsgeschichte, München 1980, hier zitiert nach Trischler: Geschichtswissenschaft Wissenschaftsgeschichte, S. 242.

17 Trischler: Geschichtswissenschaft - Wissenschaftsgeschichte, S. 242.

18 Jürgen Mittelstraß: Die Möglichkeit von Wissenschaft, Frankfurt/M. 1974, S. 114 u. 115 f.

19 Jürgen Mittelstraß: Neuzeit und Aufklärung. Studien zur Entstehung der neuzeitlichen Wissenschaft und Philosophie, Berlin, New York 1970, S. 266.

20 Vgl. dazu die Bestandsaufnahme von Stephen Shapin: Discipline and Bounding. The History and Sociology of Science as Seen Through the Externalism-Internalism-Debate, in: History of Science 30 (1992), S. 337-369. Der Beginn dieser Debatte ist umstritten. Als Beleg, dass sie nicht erst durch Kuhns „Structure of Scientific Revolutions“ ausgelöst wurde, führt Trischler exemplarisch einen Beitrag Hans Schimanks an: Edmund Hoppe oder über Inhalt, Sinn und Verfahren einer Geschichtsschreibung der Physik, in: Archiv für Geschichte der Mathematik, der Naturwissenschaft und der Technik 11 (1928/29), S. 345-351 (vgl. Trischler: Geschichtswissenschaft - Wissenschaftsgeschichte, S. 253). Eine „nicht-positivistische Konzeption“ der Wissenschaftshistoriographie sieht Canguilhem bereits durch Gaston Bachelard entwickelt (vgl. George Canguilhem: Die Geschichte der Wissenschaften im philosophischen Werk Gaston 
senschaftliche Entwicklungen aus dem Zusammenspiel politischer, ökonomischer, sozialer und ethisch-religiöser Faktoren und sieht „in der Wissenschaftsgeschichte die Erklärung eines Kulturphänomens durch die Bedingungen der gesamten kulturellen Umwelt“; ${ }^{21}$ der internalistische Ansatz hingegen bindet wissenschaftliche Phänomene vorwiegend an wissenschaftsimmanente theoretisch-methodische Probleme zurück, behandelt also „die Tatbestände der Wissenschaftsgeschichte als wissenschaftliche Tatbestände [...], was einer epistemologischen Position entspricht, welche die Theorie gegenüber der empirischen Gegebenheit privilegiert“. ${ }^{22}$ Beide Positionen bleiben notwendig defizitär: Der externalistische Ansatz reduziert Wissenschaftsgeschichte letztlich auf eine „naturalistische Soziologie der Institutionen“, der internalistische auf die „Interpretation eines Diskurses mit Wahrheitsanspruch“.23

In den letzten beiden Jahrzehnten des 20. Jahrhunderts verlagert sich der Schwerpunkt der Fragestellungen in der Wissenschaftsgeschichte „,von der kognitiven Ebene hin zur gesellschaftlich-sozialen Ebene“, ${ }^{24}$ vom „Was“ des wissenschaftlichen Gegenstands zum „Wie“ der wissenschaftlichen Forschungspraxis:

Der logische Positivismus scheint endgültig ausgedient zu haben. Die starke These lautet: Die Vorstellung einer überzeitlichen wissenschaftlichen Wahrheit und Einheit der Wissenschaft ist zutiefst ahistorisch: Wissen und Wissensproduktion sind temporal und variant..$^{25}$

Die ahistorischen Prämissen einer fortschrittsideologisch orientierten Wissenschaftsgeschichte - wissenschaftliche Theorien, abstrakte Entdeckungen und

Bachelards, in: ders.: Wissenschaftsgeschichte und Epistemologie. Gesammelte Aufsätze, hrsg. v. Wolf Lepenies, Frankfurt/M. 1979, S. 1-21, hier S. 19).

21 Canguilhem: Der Gegenstand der Wissenschaftsgeschichte, S. 28.

22 Ebd. In seinen radikaleren Ausgestaltungen entspricht der internalistische Ansatz jener oben beschriebenen ,ahistorischen“ Fortschrittsgeschichte: „In einer strikteren Version wurden dann die Ordnung und Gesetzmäßigkeit der Natur selbst sowie die universell gültigen und historisch invarianten Kategorien wie Objektivität, Rationalität und Wahrheit als Triebkräfte des wissenschaftlichen Fortschritts angesehen“ (Michael Hagner: Ansichten der Wissenschaftsgeschichte, in: Ansichten der Wissenschaftsgeschichte, hrsg. v. ders., Frankfurt/M. 2001, S. 7-39, hier S. 9). Zu den bekannteren Vertretern des externalistischen Ansatzes zählen John Desmond Bernals vierbändige Sozialgeschichte der Wissenschaften (Reinbek b. Hamburg 1970), zu den Vertretern des internalistischen Ansatzes Alexandre Koyré: Von der geschlossenen Welt zum unendlichen Universum (Frankfurt/M. 1969) oder Arthur O. Lovejoy: Die große Kette der Wesen. Geschichte eines Gedankens (Frankfurt/M. 1985).

23 Canguilhem: Der Gegenstand der Wissenschaftsgeschichte, S. 28.

24 Trischler: Geschichtswissenschaft - Wissenschaftsgeschichte, S. 242.

25 Ebd., S. 243. 
Ideen, szientifische Logik und Rationalität, Objektivität - wurden nun ihrerseits einer Historisierung und Pragmatisierung unterworfen und konnten solcherart modifiziert Eingang in die überwiegend sozialkonstruktivistisch betriebene, Wissenschaft als „Science in Action“26 begreifende Wissenschaftsgeschichte finden. Epistemologisch relevante Kategorien wie Beweis oder Naturgesetz werden nun „Gegenstand vergleichender historischer Untersuchung“, d.h. als „diskursive Formationen“ in den Blick genommen, „die sich durch verschiedene Disziplinen hindurch entwickelt und gefestigt haben“. ${ }^{27}$ Auch rücken im Zuge dieses „,experimental turn““ erstmals auch die „materiellen Repräsentationsformen der wissenschaftlichen Gegenstände und Phänomene“ sowie Fragen nach dem „Transfer von bloßen Phänomenen, Auffälligkeiten oder Daten in wissenschaftliche Erkenntnis“ in den Fokus wissenschaftsgeschichtlichen Interesses. Nicht zuletzt wird gerade in jüngerer Zeit das die wissenschaftliche Praxis (mit-)prägende, nicht explizit formalisierbare „implizite Wissen“ zum Gegenstand wissenschaftshistorischer Untersuchungen gemacht. ${ }^{28}$

Die seitens der Wissenschaftsgeschichte dominierende Auffassung von „Wissenschaft als sozialem Prozeß“ und damit einhergehend das „Erkenntnisziel einer gesellschaftshistorischen Kontextualisierung der Wissenschaften“ einerseits, die nahezu zeitgleich erfolgende „Integration sozialwissenschaftlicher [und zunehmend auch kulturwissenschaftlicher] Theorieangebote“ auch in der Geschichtswissenschaft andererseits, haben die „Voraussetzungen für eine Konvergenz von Wissenschaftsgeschichte und Geschichtswissenschaft verbessert“. ${ }^{29}$ Zugespitzt formuliert: Je weiter die wissenschaftshistorische Öffnung für eine dezidiert historische Perspektivierung ihres Gegenstandes ,Wissenschaft" fortschreitet, desto mehr wächst sie einer ihrerseits methodisch und thematisch amplifizierten Geschichtswissenschaft als deren integraler Gegenstand zu. Entsprechend hat die Geschichtswissenschaft „die Untersuchung der (Na-

26 Hagner: Ansichten der Wissenschaftsgeschichte, S. 21.

27 Hier und im Folgenden: Ebd., S. 21 u. 22.

28 Vgl. ebd., S. 23. Besondere Beachtung finden dabei Aspekte wie „Handwerk und Geschicklichkeit, körperliche Disziplin und gestisches Wissen, Aufmerksamkeit und Leidenschaften“ (ebd.). Im Lichte dieses wissenschaftshistorischen (und -theoretischen) Interesses werden vor allem auch solche philosophischen Betrachtungsweisen herangezogen, die bereits sehr früh wie etwa die Prozessphilosophie Whiteheads oder Polanyis „The Tacit Knowledge“ - das ,Irrational-Intuitive' rationaler Prozesse gewürdigt haben.

29 Trischler: Geschichtswissenschaft - Wissenschaftsgeschichte, S. 244. „Gesellschaftsgeschichte, Kulturgeschichte, Historische Anthropologie, ,linguistic turn', Mentalitätsgeschichte und Mikrohistorie stehen beispielhaft für den neuen Pluralismus der Geschichtswissenschaft in Methodik wie in Thematik: für eine ,Geschichte ohne Zentrum““ (ebd.). 
tur-)Wissenschaften im Prozeß der Modernisierung als genuin historisches Aufgabenfeld zu entdecken begonnen“..30 Im Zusammenhang mit der literarischen Wissenschaftsgeschichtsschreibung gilt es zwei Konvergenzen zwischen Wissenschaftsgeschichte und Geschichtswissenschaft besonders hervorzuheben: das von Lawrence Stone 1979 (kritisch) konstatierte „Revival of the Narrative“ und die damit einhergehende „Wiederbelebung der Biographie als historiographischen Genres““. ${ }^{31}$ Mit der ,Wiederbelebung des Narrativen ${ }^{32}$ verbindet sich bekanntermaßen nicht nur die Reflexion auf die sprachlich-erzählerischen Darstellungs-, Repräsentations- und Dokumentationsmodi der (Wissenschafts-)Historiographie selbst, sondern - dies gleichsam als Effekt einer Entwicklung, die vom linguistic turn bis zum poststrukturalistischen „Il n'y a pas de hors-texte“ (Derrida) reicht - auf dem Prüfstand stehen damit auch jene Parameter, die das ,Wissenschaftliche“ der Wissenschaft(en) konstituieren und von den Bereichen des Ästhetischen, des Ethisch-Religiösen, des lebensweltlichen Erfahrungswissens usw. definitorisch abgrenzen sollen. Problematisch erscheint insbesondere das Konzept der „Realität“ und damit die Frage nach dem Verhältnis von Text und Kontext. Unabhängig davon, welche Richtung im einzelnen bevorzugt wird - das Spektrum reicht vom dogmatischen Beharren auf der Gegebenheit einer sprachunabhängigen Wirklichkeit bis hin zur völligen In-Differenzierung von Text und Realität -, so kann bereits die schlichte Tatsache, dass man sich überhaupt vor eine Entscheidung gestellt sieht, die ernsthafte Frag- und Diskussionswürdigkeit einer vormals (zumindest mehr oder weniger) selbstverständlich gegebenen fundamentalen Verschiedenheit von Text und Wirklichkeit nicht länger verdecken. Für die Geschichtswissenschaft und Wissenschaftsgeschichte bedeutet der Abschied von den großen - auch und gerade wissenschaftliches Handeln legitimierenden - „Meta-Erzählungen“33 nicht nur eine Entlastung von ideolo-

30 Ebd., S. 244. Beispiele für diese Entwicklung ebd., S. 244 f. sowie S. 246-250.

31 Ebd., S. 248.

$32 \mathrm{Zu}$ einer ausführlichen Aufarbeitung der aktuellen Forschungsdebatte über die „Narrativität historiographischer Werke“ (S. 29) und den damit verbundenen „Konstruktcharakter von Geschichte“ (S. 30) vgl. Max Doll: Der Umgang mit Geschichte im historischen Roman der Gegenwart. Am Beispiel von Uwe Timms Halbschatten, Daniel Kehlmanns Vermessung der Welt und Christian Krachts Imperium, Frankfurt/M. 2017, bes. S. 37-42.

33 Jean-François Lyotard: Das postmoderne Wissen. Ein Bericht, übers. v. Otto Pfersmann, hrsg. v. Peter Engelmann, Graz u. Wien 1986. Vgl. hierzu einschränkend Doll unter Bezugnahme u.a. auf A. Assmann: „Trotz Abkehr von der frühzeitlichen Funktionalisierung des Vergangenen bleibt Geschichte legitimatorisch einer großen Narration verhaftet“, d.h. sie dient „zuvorderst der Legitimation von Herrschaft und der bestehenden Ordnung, wenngleich in einem weiteren, d.h. gesamtgesellschaftlichen Bezugsrahmen“ (Doll: Umgang mit Geschichte, S. 30). 
gisch oder idealistisch begründeten Geschichtsbildern, sondern vor allem die Last, ihren ureigensten Gegenstand, nämlich die ,Vergangenheit', und ihre Möglichkeiten, diesen als Vergangenes zu thematisieren und darzustellen, neu auszuloten. Thematische Umorientierungen - kultur-, geschlechter-, mentalitäts- und individualgeschichtliche Ausdifferenzierungen der Geschichtswissenschaft gehören ebenso hierher wie die vermehrte Thematisierung symbolischer, diskursiver und ,medialer' Formen der Weltaneignung -, eine verstärkte Reflexion sowohl der eigenen wissenschaftstheoretischen und -praktischen Voraussetzungen als auch des Verhältnisses zu anderen wissenschaftlichen Disziplinen sind Versuche, den mit der ,Postmoderne“ sich einstellenden Herausforderungen konstruktiv zu begegnen.

Die Deligitimierung ${ }^{34}$ der ,großen Erzählungen', d.h. ihre Entlarvung als fiktionale Mythen, hat ihre Zersplitterung in ,kleine Erzählungen' zur Folge und damit: die Pluralisierung und Fragmentierung der sozialen Lebenswelt, der Handlungs- und Wissensformen und der mit diesen jeweils verbundene Anspruch, ,wahr` zu sein. In diesem Sinne etwa ,deligitimiert‘ Hayden White ${ }^{35}$ die Geschichtswissenschaft als eine Meta-Erzählung des Vergangenen: Der Historiker „macht“ ${ }^{\text {“36 }}$ Geschichte, indem er die faktischen Bestandteile einer (ihrerseits bereits kodierten ${ }^{37}$ ) Chronik - die „Elemente einer Geschichte““38 - „als Bestandteile bestimmter Arten von Plotstrukturen“ kodiert. Erst durch dieses „fiktionsbildende Verfahren“39 des „emplotment“ werden die ursprünglich fremden, geheimnisvollen oder exotischen Ereignisse „verstehbar gemacht“40, werden ihnen Kohärenz und Bedeutung zugeschrieben. Die Art der Plotstruktur, d.h. ob historische Ereignisse „ihren Platz am Ende in einer tragischen, komischen, romantischen oder ironischen Geschichte [story] finden [...], hängt von der Entscheidung des Historikers ab, sie entsprechend den Erfordernissen der einen

34 Vgl. Lyotard: Das postmoderne Wissen, S. 112-122 sowie ders.: Memorandum über die Legitimität, in: Postmoderne und Dekonstruktion. Texte französischer Philosophen der Gegenwart. Mit einer Einführung hrsg. v. Peter Engelmann, Stuttgart 1990, S. 54-75.

35 Hayden White: Der historische Text als literarisches Kunstwerk, in: Geschichte schreiben in der Postmoderne. Beiträge zur aktuellen Diskussion, hrsg. v. Christoph Conrad u. Martina Kessel, Stuttgart 1994, S. 123-157.

36 Ebd., S. 128.

37 Vgl. ebd., S. 140.

38 Ebd., S. 127.

39 Ebd., S. 131.

40 Ebd., S. 134; zum Aspekt der Kohärenz vgl. S. 139-141. 
Plotstruktur oder des einen Mythos statt eines anderen anzuordnen“. ${ }^{41}$ Ein Ereignis wie die Französische Revolution kann demnach in verschiedenen Geschichten dargestellt werden und damit verschiedene Kohärenz- und Bedeutungsformationen annehmen. Die entmythologisierende - oder ,delegitimieren$\mathrm{de}^{6}$ - Funktion dieser von White vorgenommenen Analysen besteht gerade darin, dass sie die Unhintergehbarkeit der ,mythischen Methode“ jeder Art von Historiographie aufdecken und damit sowohl die Mythologisierung, Monumentalisierung und Heroisierung von historischen Ereignissen oder Personen als Effekt ihrer narrativen Darstellung ,outet' als auch den legitimierenden Anspruch des Historiographen, vergangene Wirklichkeit ,wahrheits'- und ,realitätsgetreu' und objektiv abbilden zu können, als epistemischen Mythos entlarvt. ${ }^{42}$ Wenn die „Narration [...] sowohl die Art und Weise [ist], wie eine Interpretation zustande kommt, als auch die Form des Diskurses, in der sich ein gelungenes Verstehen historischer Sachverhalte präsentiert“, ${ }^{43}$ dann ist das

41 Ebd., S. 129. Zum damit zusammenhängenden „Problem ästhetischer Geschichtskonsistenz“ vgl. auch Geppert: Der ,andere“ historische Roman, S. 153 ff.

42 Mit der Betonung des Narrativen und Imaginativen - „Es spielt keine Rolle, ob die Welt als real oder lediglich vorgestellt verstanden wird; die Art der Sinnstiftung (making sense) ist die gleiche“, so White in: Auch Klio dichtet oder die Fiktion des Faktischen. Studien zur Tropologie des historischen Diskurses, Stuttgart 1991, S. 121 - behauptet White jedoch keinesfalls die Identität von Fiktion und Historie; vielmehr gilt, ,dass historische Ereignisse sich von fiktionalen Ereignissen in eben der Weise unterscheiden, wie dies in der Nachfolge von Aristoteles immer wieder beschrieben worden ist. Historiker haben es mit Ereignissen zu tun, die einem bestimmten raumzeitlichen Ort zugewiesen werden können, Ereignissen, die im Prinzip beobachtbar sind oder wahrnehmbar sind (oder waren), während Autoren fiktionaler Literatur Dichter, Romanautoren, Dramatiker - es sowohl mit jener Art als auch mit vorgestellten, hypothetischen oder erfundenen Ereignissen zu tun haben“ (ebd., S. 145). Zu einer „Poetologie des historischen Wissen“ in kritischer Abgrenzung und Weiterführung von Whites' Ansatz vgl. Daniel Fulda: Poetologie des Wissens. Probleme und Chancen am Beispiel des historischen Wissens und seiner Formen (Vortrag auf dem 15. Göttinger Workshop zur Literaturtheorie am 20.06.2008), abrufbar über http://www.simonewinko.de/fulda_text.htm; letzter Zugriff: 26.08.20). Zur näheren Abgrenzung von traditioneller und postmoderner Geschichtsschreibung vgl. auch die luziden, überdies in eine ebenso prägnante wie besonnene ModernePostmoderne-Diskussion eingebetteten Ausführungen von Scholz (Gerhard Scholz: Zeitgemäße Betrachtungen? Zur Wahrnehmung von Gegenwart und Geschichte in Felicitas Hoppes Johanna und Daniel Kehlmanns Die Vermessung der Welt, Innsbruck u.a. 2012, S. 9-22. „Postmodern“, so Scholz, ,ist die Thematisierung der Historie als Fiktion und der Fiktion als Historie“ (ebd., S. 20). Ob damit Gepperts Hiatus-These obsolet geworden ist (vgl. ebd.), halte ich für fragwürdig, zumal diese von einem höchst variablen Verhältnis von Historie und Fiktion ausgeht.

43 Hayden White: Die Bedeutung der Form. Erzählstrukturen in der Geschichtsschreibung [1987], Frankfurt/M. 1990, S. 80. 
Narrative (und mit ihm das Rhetorisch-Tropische, Imaginäre, Fiktive, Ästhetische) ein Geschichte und Literatur gleichermaßen zugehöriges Konstitutionsmerkmal. ${ }^{44}$ Die Differenz zwischen Literatur und Historie lässt sich dann nurmehr durch die Analyse der für die verschiedenen „Diskursmodalitäten“ ${ }^{\text {45 }}$ jeweils „speziellen Merkmale“ und damit durch eine möglichst exakte „Vermessung des Grenzverlaufs zwischen dem Imaginären und dem Realen“ feststellen. Diese Grenze - und dementsprechend die Resultate ihrer Vermessung - ist jedoch keine ,ein für allemal' fixierbare, sondern ihrerseits dem historischen Wandel - dem je sich verändernden Blick aus der Gegenwart zurück auf die Vergangenheit - unterworfen.

Ähnliche ,deligitimierende‘ Funktionen lassen sich für das Genre der historiographischen Biographie feststellen. Wenn die „Renaissance des wirklichen Menschen“ nicht nur die „klassischen Exponenten historischer Größe“ betrifft, sondern auch die „,kleinen Leute‘, ob Dienstmädchen, Handwerker oder Fabrikarbeiter“, dann verändern und relativieren sich Status, Funktion, Bedeutung und Relevanz der großen Geschichtsträger erheblich, wie umgekehrt die ,kleinen Leute‘ allererst zu Funktoren, Trägern und ,Machern' der Geschichte avancieren. Durch die Beiordnung von ,Großem“ und ,Kleinem‘ stellt sich eine Art ,parataktischer Effekt‘ ein, der in erster Linie darin besteht, dass die Geschichte als Geschichte vorzugsweise ,großer Männer‘ als Quasi-Fiktion entlarvt wird. Wie in der Geschichtswissenschaft hat sich auch in der naturwissenschaftlichen Hagiographie ${ }^{46}$ eine „subjektive Wende“447 vollzogen, die das „traditionel-

44 Das Narrative ist so betrachtet nicht länger der exklusive Darstellungsmodus fiktiver Literatur, sondern eine allgemeine Weltzugangs- und Weltdarstellungsweise, deren Status auch außerhalb ihres spezifisch literarischen Gebrauchs zu diskutieren ist. $\mathrm{Zu}$ einer allgemeinen Erzähltheorie vgl. Albrecht Koschorke: Wahrheit und Erfindung. Grundzüge einer Allgemeinen Erzähltheorie, Frankfurt/M. 2012.

45 Hier und im Folgenden White: Die Bedeutung der Form, S. 76 f., 62.

$46 \mathrm{Vgl}$. auch Hagner, der die Aufteilung in eine externalistische und internalistische Wissenschaftsgeschichte durch den Umstand ergänzt wissen möchte, „dass die Geschichte der Wissenschaften zu keinem Zeitpunkt ohne ein heroisches Verständnis des wissenschaftlichen Fortschritts zu haben gewesen ist. Kategorien wie Genius, Kreativität und Intuition sind hier zum Zuge gekommen.“ In diesem Zusammenhang ist auch die wissenschaftliche Biographie zu sehen: „Die unpersönliche Welt der rationalen Strukturen und Systeme auf der einen Seite und die leidenschaftliche Welt von individueller Kreativität und Ingeniosität auf der anderen standen keineswegs in permanenter Auseinandersetzung um den richtigen Weg einer Wissenschaftsgeschichtsschreibung. Vielmehr haben beide ihren jeweiligen Geltungsbereich gefunden, indem sie an ein unterschiedliches Publikum adressiert sind und sich in verschiedenen Textgenres ausdrücken“ (Hagner: Ansichten der Wissenschaftsgeschichte, S. 10 f.). Mit der 
le Konzept wissenschaftlicher Größe und Genialität“ verabschiedet, indem sie „Individualität und Sozialität mit dem Instrumentarium personenzentrierter Forschung“ verknüpft und dabei vor allem die „Frage nach den Handlungs- und Entscheidungsspielräumen von Individuen in systemischen Kontexten“ berücksichtigt. „Bringing the Human Actors Back on Stage“ ${ }^{48}$ - mit dieser Formulierung eines amerikanischen Wissenschaftshistorikers ist die Tendenz der Rückbindung einer den ,menschlichen Faktor ${ }^{*}$ eliminierenden, objektivistischen Auffassung von Wissenschaft an ihren humanen und kulturellen Ursprung treffendbezeichnet.

subjektiven Wende hingegen treten Leben und Wissenschaft, Biographie und Wissenschafts(geschichts)schreibung in ein Interdependenzverhältnis.

47 Hier und im Folgenden Trischler: Geschichtswissenschaft - Wissenschaftsgeschichte, S. 249.

48 David Kaiser: Bringing the Human Actors Back on Stage. The Personal Context of the Einstein-Bohr Debate, in: British Journal for the History of Science 27 (1994), S. 129-152. 